\title{
Exposure Time Point
}

National Cancer Institute

\section{Source}

National Cancer Institute. Exposure Time Point. NCI Thesaurus. Code C83157.

A point in time that the exposure takes place. 